\title{
Análise dos investimentos na capacidade de inovar nos indicadores de risco das empresas brasileiras
}

\section{Analysis of investments in the ability to innovate on risk indicators of Brazilian companies}

\author{
Vitor Novelini Belotti Graduado em Administração, Ernest \& Young Global Consulting Service (EY), \\ Brasil - vitor_belotti@hotmail.com \\ David Ferreira Lopes Santos Doutor em Administração de Empresas, Universidade Estadual Paulista "Júlio \\ de Mesquita Filho" (Unesp), Brasil - david.lopes@unesp.br
}

\section{RESUMO}

O investimento na capacidade de inovar tem se tornado uma estratégia recorrente por empresas que buscam liderar o mercado em que atuam. Entretanto, todo investimento também é composto pelo risco, que tende a direcionar e auxiliar os interessados em seu possível retorno. Assumir riscos e mensurar as incertezas decorrentes das decisões tomadas é uma tarefa própria ao investidor, que se guiará através de indicadores para tomar a decisão considerada correta em determinado momento. O objetivo deste trabalho é avaliar o impacto dos investimentos na capacidade de inovar nos indicadores de risco das 146 maiores empresas brasileiras listadas na B3 (Brasil - Bolsa - Balcão), no período de 2012 a 2015, período caracterizado por fortes questões políticas e econômicas. A partir de uma abordagem quantitativa, composta por indicadores de inovação e de risco, e com base no modelo de regressão linear com dados em painel, verificou-se que as variáveis não seguem um padrão linear, ora apresentando determinada relação, seja ela positiva ou negativa, ora o contrário. O fato pode ser decorrente da dispersão dos dados analisados ou das contingências presentes no ambiente em que cada empresa está inserida, visto que o período analisado foi de grande instabilidade política e econômica, influenciando negativamente no cenário organizacional.

Palavras-chave: Investimento em Inovação. Finanças de longo prazo. Desempenho econômico-financeiro. Regressão com dados em painel.

\section{ABSTRACT}

Investment in the ability to innovate has become a recurrent strategy for companies that seek to lead the market where they operate. However, all investment is also composed by risk that tends to direct and assist stakeholders in its possible return. Taking risks and measuring the uncertainties resulting from the decisions taken is an innate task for the investor who will be guided through indicators to make the decisions considered correct at a given moment. The objective of this study is to evaluate the impact of investments in the ability to innovate on the risk indicators of the 146 largest Brazilian companies listed in B3 (Brasil Bolsa Balcão) in the period from 2012 to 2015, a period characterized by strong political and economic issues. Based on the quantitative approach, composed of innovation and risk indicators, and based on the linear regression model with panel data, it was verified that the variables don't follow a linear pattern, at times presenting a certain relation, either positive or negative, and sometimes the opposite. This fact can be due to the dispersion of analyzed data or the present contingencies in the environment in which each company is inserted as the analyzed period was of a great political and economic instability influencing adversely in the organizational scenery.

Keywords: Investment in innovation. Long-term finance. Economic and financial performance. Regression with panel data.

Recebido em 03/06/2019. Aprovado em 04/08/2019. Avaliado pelo sistema double blind peer review. Publicado conforme normas da ABNT. http://dx.doi.org/10.22279/navus.2020.v10.p01-16.991 


\section{INTRODUÇÃO}

A busca pela liderança de mercado, a partir do investimento em inovações, está cada vez mais recorrente no contexto das organizações, devido ao alto grau de competitividade presente nos diversos mercados, visto que através de um diferencial criado pela empresa, esta pode alcançar uma posição de destaque no mercado de forma mais fácil (CASSOL; ZAPALAI; CINTRA, 2017).

A inovação é considerada um fator essencial para obtenção de vantagem competitiva e por isso, as empresas investem em recursos que permitam a geração de inovações, tais como: Pesquisa e Desenvolvimento (P\&D), Aquisição de novas máquinas e equipamentos, Aquisição de conhecimento externo, entre outros (BESANKO et al., 2006; GODIN, 2017). Santos, Basso e Kimura (2012) entendem que a inovação é um processo e, portanto, os resultados só ocorrem mediante "inputs" (humanos e de capital), além de uma gestão desses recursos alinhadas à estratégia empresarial. A estrutura desses recursos orientados à inovação pode ser denominada como capacidade de inovação (CROSSAN; APAYDIN, 2010; SANTOS; BASSO; KIMURA, 2012).

O entendimento da capacidade de inovar como um recurso, tem seus fundamentos da Teoria Baseada em Recursos, que classifica esse recurso como intangível e, assim como outros intangíveis há uma dificuldade teórica em estudá-lo em função de limitações metodológicas e das diferentes variáveis com as quais se relacionam (BARNEY; WRIGHT; KETCHEN, 2001).

A capacidade da empresa para inovar é resultado dos investimentos feitos por ela em resposta aos estímulos causados pelos ambientes internos e externos em que ela está inserida (COELHO; CAMARA; BRASIL, 2014), sendo definida a partir das experiências e dos conhecimentos adquiridos no exercício das atividades da firma e responsáveis por diferenciá-la e impulsioná-la (RAMOS; ZILBER, 2015).

Diferentes pesquisadores, usando métodos quantitativos e qualitativos, com múltiplas abordagens, têm estudado a relação entre inovação e desempenho (JIMÉNEZ-JIMÉNEZ; SANZ-VALLE, 2011; PRAJOGO, 2016). Porém, não existe consenso teórico sobre o efeito do investimento em inovação no desempenho empresarial (SANTOS; BASSO; KIMURA, 2018). Um dos fatores que contribuem para discordância teórica existente na relação entre inovação e desempenho é o risco associado a estes investimentos (SHAFIQUE, 2013; ETGES; CORTIMIGLIA, 2019).

Os investimentos em inovação, assim como qualquer investimento, são caracterizados pela expectativa de retorno futuro. Entretanto, a possibilidade de variações desse retorno é o que caracteriza o risco do investimento e de qualquer recurso organizacional, sendo necessário que a firma identifique os riscos e tenha ações para gerenciar os possíveis efeitos negativos ou não desejados que os resultados podem reportar (MATTA; OLIVA; VASCONCELLOS, 2015; OSORIO et al., 2019).

O risco dos investimentos em inovação é decorrente da incerteza quanto ao retorno desses gastos, tendo em vista, a natureza incerta dos processos de inovação no que diz respeito à eficácia (geração de novos produtos ou processos) e ao tempo em que esses resultados ocorrerão (SANTOS; BASSO; KIMURA, 2018).

Por outro lado, paradoxalmente, os investimentos em inovação podem auxiliar na redução do risco das empresas junto ao mercado, pois a realização de investimentos com essa finalidade pode sinalizar que haverá benefícios de longo prazo aumentando a atratividade da empresa e reduzindo a sua volatilidade (BASSO; KIMURA, 2010).

Por isso, existe a necessidade em analisar a influência dos investimentos nos recursos destinados à inovação no risco da empresa, pois o aumento do risco pode refletir na redução do valor de mercado das empresas, na redução das linhas de crédito e no maior comprometimento do caixa com o pagamento de despesas financeiras em razão do aumento das taxas de juros (AMORIM; LIMA; MURCIA, 2012).

A literatura tem evidenciado o risco inerente aos investimentos na capacidade de inovar das empresas podendo ser endógenos e/ou exógenos às firmas, constituindo-se como um desafio para acadêmicos e profissionais de mercado que procuram modelá-los (WU et al., 2010; ILEVBARE; PROBERT; PHAAL, 2014; SANTOS; BASSO; KIMURA, 2018; ETGES; CORTIMIGLIA, 2019).

Desse modo, esse estudo procura auxiliar na melhor compreensão a respeito do relacionamento entre os investimentos na capacidade de inovar e o risco das empresas. A formalização da motivação para o 
desenvolvimento desse estudo foi: Qual o grau de impacto entre inovação e risco no cenário organizacional? Diante disso, esta pesquisa teve como objetivo: Avaliar o impacto dos investimentos na capacidade de inovar nos indicadores de risco de empresas brasileiras no período de 2012 a 2015, período de grande instabilidade política e econômica, principalmente no contexto nacional.

No intuito de atingir os objetivos propostos, este trabalho segue com a seção Referencial Teórico que sustenta as principais premissas presentes nessa pesquisa: inovação e risco; na sequência, são abordados os materiais e métodos utilizados neste estudo; e em seguida resultados da pesquisa, acompanhada das discussões. Por fim, a última seção traz as considerações finais com destaque para as implicações, conclusões e limitações.

\section{REFERENCIAL TEÓRICO}

Em razão do objetivo desse estudo, buscou-se na literatura - sobre inovação e risco - as bases teóricas para construir o modelo empírico e analisar os resultados alcançados. Salienta-se que, nesse estudo utiliza-se a base teórica da Visão Baseada em Recursos que compreende a inovação como um ativo intangível da empresa, cujos resultados decorrem de investimentos e gestão em uma estrutura de recursos que promova inovações (CROSSAN; APAYDIN, 2010; SANTOS; BASSO; KIMURA, 2018).

\subsection{Capacidade de inovar}

Fator indispensável para alavancar o crescimento das organizações, a inovação tem assumido uma posição de destaque no planejamento estratégico das organizações (BESANKO et al., 2006; RAMOS; ZILBER, 2015). As expectativas estratégicas com a inovação são variadas entre as empresas, destacam-se: acelerar a abertura de novos mercados; criar novos produtos que atendam às necessidades dos consumidores de forma personalizada; aumentar a sustentabilidade dos processos; entre outros (THEISS et al., 2014; GODIN, 2017).

Para que os objetivo pretendidos com a inovação sejam efetivos, as empresas devem ser capazes de inovar, tendo em vista, que não basta estabelecer a inovação como uma estratégia e não ter condições internas ou externas para realizá-la (PRAJOGO, 2016). Como explicam Crossan e Apaydin (2010), a inovação é um processo que requer investimentos em "inputs" necessários à formação de um recurso organizacional capaz de gerar inovações. Esse recurso organizacional tem sido estruturado na literatura como capacidade de inovar (SANTOS; BASSO; KIMURA, 2018).

Entender a inovação como um processo é fundamental do ponto de vista conceitual, para que as decisões empresariais não sejam tomadas de forma equivocada (CROSSAN; APAYDIN, 2010). Por exemplo, Schumpeter (1927, p. 295, tradução nossa) conceitua:

Entendo a inovação como mudanças nas combinações dos fatores de produção que não podem ser efetuadas por etapas infinitesimais ou variações marginais. As inovações consistem principalmente em mudanças nos métodos de produção $e$ transporte, ou em mudanças na organização industrial, ou na produção de um novo produto, ou na abertura de novos mercados ou de novas fontes de material.

Nota-se nesta definição que a inovação é retratada pelos seus resultados, isto é, aquilo que se espera com a inovação. No entanto, os resultados de inovação não ocorrem de forma aleatória e, tampouco, são obtidos sem uma estrutura que os viabilize, ou seja, empresas inovadoras precisam ter um aparato institucional e estrutural que envolve pessoas, máquinas, equipamentos, materiais, serviços entre outros inputs que contribuam à construção de uma capacidade de inovar (CROSSAN; APAYDIN, 2010; GODIN, 2017; OSORIO et al., 2019).

Diante disso, há a necessidade de uma política de investimentos na capacidade de inovar, cujo planejamento é de responsabilidade da gestão da empresa e que poderá assumir uma característica 
conservadora (pouco investimento e paralisação do crescimento devido à atitude de resguardo do caixa) ou agressiva (elevado investimento e possível influência positiva no desempenho empresarial), sendo uma decisão relevante para a empresa e determinada por fatores internos e externos (BAÑOS-CABALLERO; GARCÍATERUEL; MARTÍNEZ-SOLANO, 2014).

A estratégia de inovação depende de diversos fatores, como: características do setor, oportunidades tecnológicas existentes e percebidas pelas organizações, acumulação anterior de conhecimento, entre outros aspectos (MENEZES et al., 2011). Vários estudos reconhecem o recurso inovação como uma variável dependente e não como um mediador para o desempenho, em que a capacidade de inovar influencia de forma favorável no desempenho da organização (CROSSAN; APAYDIN, 2010).

Pang et al. (2019) evidenciam no seu estudo bibliométrico que dependendo da forma como as organizações atuam no mercado, elas garantiriam uma menor volatilidade ou um maior rendimento de seus ativos. No entanto, esse fato também pode refletir diferenças entre os setores industriais (concentração, nível de competitividade, regulação, entre outros) e as características dos stakeholders e shareholders (empresas familiares, pulverização de controle acionários, relacionamento com ONGs, entre outros).

Diante da complexidade que envolve a análise dos investimentos na capacidade de inovar das empresas, ainda há uma lacuna quanto aos métodos de aferição do risco do recurso inovação (BASSO; KIMURA, 2010). Em que pese a dificuldade metodológica, os investimentos em inovação, assim como qualquer outro investimento, devem reportar um retorno compatível com o seu risco (OSORIO et al., 2019).

$O$ risco dos investimentos na capacidade de inovar pode ser compreendido pela variabilidade de resultados possíveis (SANTOS; BASSO; KIMURA, 2018). Por outro modo, investir na capacidade de inovar não garante que a empresa conseguirá os resultados esperados ETGES; CORTIMIGLIA, 2019. Há a possibilidade de gerar inovações que modifiquem paradigmas tecnológicos ou permitam a criação de vantagem competitiva, como também, existe a possibilidade dos esforços para desenvolver essa capacidade de inovar não gerar resultados significativos e, com efeito, a empresa terá que amortizar o investimento realizado e subsidiar as consequências (perda de liquidez, redução de rentabilidade, possíveis prejuízos, perda de mercado, entre outros) (BAÑOS-CABALLERO; GARCÍA-TERUEL; MARTÍNEZ-SOLANO, 2014; ETGES; CORTIMIGLIA, 2019).

Diante disso, a necessidade em avaliar o risco dos investimentos orientados a capacidade de inovar é relevante, porém a mensuração do risco de ativos empresariais também é um dos principais desafios na teoria das finanças corporativas (DANTHINE; DONALDSON, 2005). A dificuldade teórica reside na seleção de indicadores e modelos capazes de capturar o risco de investimentos, principalmente, quando os retornos não podem ser dimensionados com clareza, em razão de incertezas tecnológicas, comerciais, logísticas, além de variáveis exógenas à firma (MARCELINO-SÁBADA et al., 2014).

Sendo assim, a fim de alcançar o propósito este estudo, estende-se a literatura até as ferramentas relacionadas ao risco, como métodos estatísticos e indicadores, que tendem a facilitar a compreensão da relevância da presente pesquisa.

\subsection{Risco}

A literatura clássica de finanças retrata o risco de um ativo como a variância de retornos esperados (DANTHINE; DONALDSON, 2005; DAMODARAN, 2010). Ressalta-se, também, o conceito de risco sistemático de um ativo que reflete, somente, a parcela do risco não diversificável de um ativo (risco de mercado determinada por fatores econômicos, políticos e sociais) (SANTOS; FONTES, 2011). Sendo a parte sistemática capaz de ser encontrada através do coeficiente Beta ( $\beta$ ), que mede o grau de influência das variações do mercado em relação ao ativo financeiro ou real (DANTHINE; DONALDSON, 2005; DAMODARAN, 2010; AMORIM; LIMA; MURCIA, 2012).

Nessa direção, a possibilidade de variações no retorno do investimento é o que caracteriza o risco do recurso organizacional, sendo esta variação, distinta entre os diferentes setores da economia em função da complexidade tecnológica que cerca cada indústria e a exposição às variáveis externas que integram o risco sistemático de cada país (BOWERS; KHORAKIAN, 2014). 
A inovação, cujo investimento é caracterizado por incertezas endógenas e exógenas às firmas, se apresenta como um desafio atual para acadêmicos e profissionais de mercado envolvidos em processos e pesquisas de Valuatione Project Finance (WU et al., 2010; ILEVBARE; PROBERT; PHAAL, 2014); sendo o elemento risco, um dos fatores determinantes no desempenho de projetos de investimento realizado pelas organizações (MIORANDO; RIBEIRO; CORTIMIGLIA, 2014).

Entretanto, quando se estuda o conceito "risco" associado a investimentos em inovação, torna-se necessário sua classificação em: tecnológico, criação própria de um fator novo no mercado (SANTOS; FONTES, 2011); empresarial, associado às estratégias e políticas internas da empresa (MARTIN; SANTOS; DIAS FILHO, 2004); e financeiro, foco deste estudo e que se refere às flutuações de variáveis financeiras que afetam negativamente o planejamento e as projeções de um projeto, trazendo altos índices de incerteza e tornando mais importante o uso de indicadores capazes de auxiliar os gestores na tomada de decisão ao investir (ETGES; CORTIMIGLIA, 2019).

O risco deve ser mensurado, já que se caracteriza como um fator determinante para os investidores que exigirão uma taxa de retorno maior se o risco for elevado e, menor, se o investimento garantir um baixo risco (DAMODARAN, 2010). Uma das alternativas para contornar esse cenário, consiste na utilização de indicadores de risco associados a investimentos feitos em inovação, já que através deles seria possível identificar o resultado ou o desempenho do investimento realizado (BASSO; KIMURA, 2010).

Todavia, a literatura que avalia a relação entre risco empresarial e os investimentos na capacidade de inovar ainda é recente e baseada a partir de questionários com executivos (MEROÑO-CERDÁN; LÓPEZNICOLÁS; MOLINA-CASTILLO, 2018). O uso das informações financeiras disponibilizadas pelas empresas para esse fim, ainda é limitada, como por exemplo o estudo de Jafari, Chadegani e Biglari (2011) que usaram as informações financeiras para os investimentos em inovação (gastos com P\&D) e utilizaram como proxy para o risco a variância das receitas.

Diversos estudos se propuseram a identificar a relação entre as informações contábeis relativas aos investimentos das empresas e o risco empresarial (mensurado pela variância dos retornos das ações ou pelo beta), sendo que os resultados são difusos, conforme apontam Amorim, Lima e Murcia (2012). É provável que a dificuldade em estabelecer uma relação entre os investimentos com o risco deve-se a heterogeneidade dos ativos, como apontam Santos e Silva (2009), como também, as diferentes formas de mensurar o risco (AMORIM, LIMA e MURCIA, 2012). Não obstante, em nenhum dos estudos verificados por esses autores, houve discriminação dos investimentos voltados à inovação.

Considerando a base de dados SCOPUS e o uso dos termos "Innovation", "Investment" and "Risk", verificou-se somente 9 artigos (pesquisa em maio/2018), sendo que nenhum abordou a relação entre os investimentos na capacidade de inovar e o risco das empresas. Assim, entende-se que a proposta desse estudo tem a originalidade em avaliar uma relação teórica já conhecida que é o risco de investimentos, porém sem uma avaliação mais ampla e direcionada para os investimentos na capacidade de inovar das empresas.

Em adição à relevância teórica, o Manual de Oslo também tem refletido essa preocupação ao relatar "Aceita-se atualmente que o desenvolvimento e a difusão de novas tecnologias são essenciais para o crescimento da produção e aumento da produtividade. Nosso entendimento do processo de inovação e seu impacto econômico ainda é deficiente" (OCDE, 2005, p. 15, grifo nosso). Por isso, não se trata, apenas de relevância teórica, mas também empírica, em melhor compreender a relação entre os investimentos no processo de inovação e o risco.

\section{MATERIAL E MÉTODOS}

Trata-se de uma pesquisa de natureza descritiva com finalidade teórica-aplicada, a partir de uma abordagem metodológica quantitativa. A escolha descritiva com finalidade teórica-aplicada deve-se ao objetivo do estudo que se propõe a avaliar a relação entre os investimentos na capacidade de inovar no risco das empresas. Assim, como já é uma relação existente, busca-se descrevê-la a partir de uma abordagem metodológica distinta dos estudos já realizados e apontados por exemplo no levantamento bibliométrico de 
Etges e Cortimiglia (2019), o que justifica a finalidade teórica e também aplicada por buscar os dados em empresas (VERGARA, 2015).

Baseando-se no objetivo propostos, o conjunto de dados necessários para essa pesquisa compreende os dados financeiros das 146 maiores empresas brasileiras, em representatividade econômica, listadas na B3, a respeito dos gastos realizados com a capacidade de inovar apontados nos relatórios de gestão das empresas da amostra (em específico, contas imobilizado e intangível, referente aos investimentos em novos produtos, novos processos, marcas e patentes, entre outros itens). Estes dados foram coletados por meio do Sistema Economática ${ }^{\circ}$, organizados em planilhas do software Microsoft Excel ${ }^{\circ} \mathrm{e}$, posteriormente, submetidos no software Gretl (Gnu Regression, Econometrics and Time-series Library, versão 2017).

Além disso, a segunda etapa da pesquisa compreende a mensuração de indicadores de risco, através dos valores das ações das empresas listadas na bolsa, durante o período de 2012 a 2015 . O período da análise foi escolhido de forma intencional com o objetivo de tornar este estudo mais atualizado, já que quando se discute investimentos em inovação torna-se essencial a utilização de dados mais recentes.

O ativo livre de risco utilizado neste trabalho para representar o retorno esperado do mercado é o CDI (Certificado de Depósito Interbancário), visto que é o mais usado em estudos que envolvem o cálculo dos indicadores listados (ARAÚJO; OLIVEIRA; SILVA, 2012). As variáveis que foram utilizadas para a análise estão listadas no Quadro 1 e foram divididas em variáveis de inovação e variáveis de risco:

Quadro 1 - Variáveis de inovação e risco

\begin{tabular}{|c|c|}
\hline Variáveis de Inovação & Variáveis de Risco \\
\hline Gasto Total com Inovação $=\ln \left(\sum\right.$ Gastos Inovação $\left.o_{i, t}\right)$ & Risco: $\sigma=\sqrt{\frac{1}{n-1} \sum(x-\bar{x})^{2}}$ \\
\hline Investimento em Inovação $=\frac{\text { Gastos com Inovação }_{i, t}}{\text { Receita }_{i, t}}$ & Beta: $\beta=\operatorname{Cov} \frac{(r a, r p)}{\operatorname{Var}(r p)}$ \\
\hline Imobilizado $=\frac{\Delta \text { Imobilizado }_{i, t}}{\text { Receita }_{i, t}}$ & Índice de Treynor: $\mathrm{IT}=\frac{R i-R f}{\beta i}$ \\
\hline Intangível $=\frac{\Delta \text { Intangível }_{i, t}}{\text { Receita }_{i}, t}$ & Índice de Sharpe: IS $=\frac{R i-R f}{\sigma i}$ \\
\hline
\end{tabular}

Fonte: Elaborado pelos autores

O beta representa o risco de mercado sendo de fundamental importância a escolha correta dos itens necessários para seu cálculo: índice de mercado, intervalo de tempo e a frequência dos dados; onde: ra é o retorno do ativo e rp é o retorno do portfólio (DAMODARAN, 2010).

O Índice de Treynor é um indicador da relação risco/retorno usado para medir o excesso de retorno conseguido por unidade de risco de mercado (risco este que não pode ser controlado pelo investidor), onde "Ri" é o retorno da empresa e "Rf" é o Risk Free ou Retorno Livre de Risco; enquanto o índice de Sharpe representa o quão bem posicionado está aquele ativo (SILVA; SANTOS; RODRIGUES, 2016).

Para construir os resultados da pesquisa e analisá-los, foram utilizadas as seguintes técnicas: medidas de posição (média), medidas de dispersão (desvio-padrão) e medidas de associação (correlação) contidas no Quadro 2; além de variáveis de risco, como: Beta, Índice de Treynor e Índice de Sharpe Ajustado, reunidas no Quadro 1.

Quadro 2 - Medidas Estatísticas

\begin{tabular}{|l|l|l|}
\hline \multicolumn{3}{|c|}{ Medidas Estatísticas } \\
\hline Média: $\bar{x}=\frac{\sum x}{n} \quad$ & Desvio-padrão: $\sigma=\sqrt{\frac{1}{n-1} \sum(x-\bar{x})^{2}}$ & Correlação: $p_{(x, y)}=\frac{\sum x_{i} y_{i}-n \bar{x} \bar{y}}{\sqrt{\left(\sum x_{i}^{2}-n \bar{x}^{2}\right)\left(\sum y_{i}^{2}-n \bar{y}^{2}\right)}}$ \\
\hline
\end{tabular}

Fonte: Elaborado pelos autores (2019).

Através da primeira fórmula chega-se à média dos investimentos realizados com inovação, e através do desvio-padrão é possível verificar o risco associado à incerteza do fluxo de investimentos em inovação 
realizados pela empresa, através dos gastos feitos durante determinado período. Já a correlação tem o papel de verificar a associação entre determinadas variáveis com o intuito de identificar o grau de interdependência entre elas, onde: 1 (associação alta e positiva), -1 (associação alta e negativa) ou 0 (sem associação).

Foi utilizado o modelo de regressão linear com os dados em painel que segue uma determinada amostra de dados ao longo do tempo e possibilita diversas observações, inclusive se a relação entre as variáveis sofre alguma mudança durante um longo período, fornecendo assim múltiplas observações sobre cada variável na amostra (HSIAO, 1986).

A análise feita com dados em painel oferece informações estatisticamente importantes para uma pesquisa científica através da maior credibilidade oferecida por seu rigor metodológico, tendo como vantagem a capacidade de controlar os efeitos das variáveis não observadas (HAIR Jr. et al., 2005).

$O$ modelo de regressão linear com dados em painel segue a estrutura seguinte, onde $Y$ representa a variável dependente (variáveis de risco), a é a constante ou intercepto, $\beta$ é o coeficiente angular da reta, $X$ representa a variável independente (variáveis de inovação), $i$ representa a unidade de corte transversal (empresa), $t$ o período de tempo e $\varepsilon$ representa os erros aleatórios da medição (Equação 7):

$$
Y_{i t}=\alpha_{i}+\beta_{1 .} X_{1 i, t}+\ldots+\beta_{n .} X_{n i, t}+\varepsilon_{i, t}
$$

Primeiramente, seria utilizado o método de regressão linear com efeitos fixos, entretanto, à medida que os cálculos foram feitos, notou-se uma forte dispersão dos dados (heterocedasticidade) ao redor da reta da equação e por esta razão, houve uma mudança do método com efeitos fixos para o método dos mínimos quadrados ponderados, que resolveu a limitação da heteroscedasticidade. O método de análise com efeitos aleatórios não foi considerado, pois como se trata de uma análise ao nível das firmas, entende-se que não é possível considerar que as decisões referentes as variáveis independentes sejam "aleatórias".

Para avaliar o ajuste do modelo foram utilizados: Coeficiente de Determinação (ou $\mathrm{R}^{2}$ que mede o grau de confiabilidade da regressão); Estatística $\mathrm{F}$ do modelo empírico (estatística capaz de mostrar se o modelo está corretamente especificado através aceitação ou rejeição de hipóteses); Autocorrelação ou Durbin-Watson (análise de correlação para modelos de regressão com efeitos fixos); e Homocedasticidade (Teste Wald), conforme pressupõe Gujarati (2006).

\section{RESULTADOS}

Com o objetivo de identificar o comportamento das variáveis inovação e risco, a

Tabela 1 compreende os resultados estatísticos descritivos das variáveis analisadas, destacando-se a média e o desvio-padrão do investimento com inovação realizado pelas empresas da amostra durante o período de 2012 a 2015, onde é possível afirmar que todas as empresas investiram em média 7,45\% da receita líquida.

Tabela 1- Estatísticas Descritivas da amostra pesquisada

\begin{tabular}{cccccc}
\hline Variáveis & Média & Mediana & Desvio-padrão & Mín. & Máx. \\
\hline Gasto Total com Inovação (\$ mil) & 791.000 & 81.400 & 2.710 .000 & 0 & 31.800 .000 \\
Gasto Total Inovação / Receita & 0,0745 & 0,02 & 0,14 & 0 & 1 \\
Variação Imobilizado & $-0,09$ & 0 & 1,54 & $-31,80$ & 2,03 \\
Variação Intangível & 0,01 & $-0,0005$ & 0,28 & $-2,78$ & 2,43 \\
Beta & 0,59 & 0,52 & 2,10 & $-10,60$ & 8,71 \\
Treynor & $-5,76$ & 0 & 191 & -2400 & 1530 \\
Risco Anual Médio & 22,92 & 27,09 & 18,51 & 0 & 99,07 \\
Sharpe & $-0,24$ & 0 & 0,70 & $-2,32$ & 2,26 \\
\hline
\end{tabular}

Fonte: Elaborado pelos autores (2019). 
Além das estatísticas descritivas, através da análise do coeficiente de correlação é possível observar o grau de associação entre as variáveis de inovação e risco, dados presentes na Tabela 2.

Tabela 2 - Matriz de correlação das variáveis do estudo

\begin{tabular}{|c|c|c|c|c|c|c|c|c|c|}
\hline Beta & Treynor & $\begin{array}{l}\text { Risco } \\
\text { Médio }\end{array}$ & Sharpe & $\begin{array}{l}\text { Gasto Total } \\
\text { Inovação }\end{array}$ & $\begin{array}{l}\text { Gasto } \\
\text { Total / } \\
\text { Receita }\end{array}$ & $\begin{array}{l}\text { Variação } \\
\text { Imobilizado }\end{array}$ & $\begin{array}{l}\text { Variação } \\
\text { Intangível }\end{array}$ & $\begin{array}{l}\text { LnGasto } \\
\text { Total } \\
\text { Inovação }\end{array}$ & \\
\hline \multirow[t]{9}{*}{1} & $-0,0147$ & 0,2238 & $-0,162$ & $-0,1434$ & $-0,0236$ & 0,0018 & 0,0537 & $-0,0813$ & Beta \\
\hline & 1 & $-0,003$ & 0,0042 & 0,0549 & $-0,0317$ & 0,0003 & $-0,0888$ & 0,0568 & Treynor \\
\hline & & 1 & $-0,149$ & 0,1045 & $-0,1177$ & 0,0656 & 0,0171 & 0,1036 & Risco Anual \\
\hline & & & 1 & 0,0712 & 0,0562 & $-0,0066$ & 0,1212 & 0,1489 & Sharpe \\
\hline & & & & 1 & 0,3148 & 0,0137 & 0,0483 & 0,637 & $\begin{array}{l}\text { Gasto Total } \\
\text { Inovação }\end{array}$ \\
\hline & & & & & 1 & $-0,0413$ & 0,1922 & 0,5095 & $\begin{array}{l}\text { Gasto Total / } \\
\text { Receita }\end{array}$ \\
\hline & & & & & & 1 & $-0,0163$ & $-0,0282$ & $\begin{array}{l}\text { Variação } \\
\text { Imobilizado }\end{array}$ \\
\hline & & & & & & & 1 & 0,1014 & $\begin{array}{l}\text { Variação } \\
\text { Intangível }\end{array}$ \\
\hline & & & & & & & & 1 & $\begin{array}{l}\text { LnGasto } \\
\text { Inovação }\end{array}$ \\
\hline
\end{tabular}

Fonte: Elaborado pelos autores (2019).

Em razão da ausência de homocedasticidade entre as empresas ao longo do tempo e autocorreção pelos testes de Wald e Durbin-Watson utilizou-se o modelo de regressão com dados em painel pelo método de mínimos ponderados.

Desse modo, após a mudança para o método dos mínimos quadrados ponderados, foi possível obter os resultados da Tabela 3, que apresentou um modelo com a mesma variável dependente anterior, sendo possível identificar que, a constante e as variáveis gasto total com inovação sobre receita e log-gasto total com inovação influenciam diretamente no beta sob qualquer possibilidade, devido a orientação similar dos intervalos de confiança e coeficientes.

Tabela 3 - Resultados da Regressão para Beta

\begin{tabular}{|c|c|c|c|c|}
\hline \multicolumn{5}{|c|}{ Usando 253 observações / 134 unidades de corte transversal } \\
\hline \multicolumn{5}{|c|}{ Variável dependente: BETA } \\
\hline & Coeficiente & Grau de Significância & $\begin{array}{c}\text { Intervalo } \\
\mathrm{T}(248\end{array}$ & $\begin{array}{l}\text { nça }(95 \%) \\
1,970\end{array}$ \\
\hline Constante & 2,4998 & $* * *$ & 1,9726 & 3,0269 \\
\hline Gasto Total com Inovação / Receita & 0,2360 & ** & 0,0479 & 0,4242 \\
\hline Variação Imobilizado & 0,0056 & - & $-0,0075$ & 0,0188 \\
\hline Variação Intangível & 0,2508 & - & $-0,1671$ & 0,6687 \\
\hline Log-Gasto Total com Inovação & $-0,1366$ & *** & $-0,1790$ & $-0,0942$ \\
\hline R-quadrado & \multicolumn{4}{|c|}{0,1503} \\
\hline Estatística F $(4,248)$ & \multicolumn{4}{|c|}{10,9698} \\
\hline P-valor (F) & \multicolumn{4}{|c|}{0} \\
\hline
\end{tabular}

Nota: ****** * significam nível de significância de 99\%, 95\% e 90\%, respectivamente Fonte: Elaborado pelos autores (2019).

As tabelas seguintes apresentam os resultados do modelo empírico para as variáveis dependente: Índice de Treynor (Tabela 4), Risco Anual Médio (Tabela 5) e Índice de Sharpe (Tabela 6), sendo possível verificar como as variáveis de inovação se comportam de maneira diferente dependendo da variável de risco analisada, ora apresentando influência de comportamento, ora não apresentando. 
Tabela 4 - Resultados da Regressão com dados em painel para Treynor Usando 253 observações / 134 unidades de corte transversal

\begin{tabular}{lcccc}
\hline & Variável dependente: TREYNOR & \\
\hline & Coeficiente & Grau de Significância & $\begin{array}{c}\text { Intervalo de Confiança (95\%) } \\
\text { T }(248 ; 0,025)=1,970\end{array}$ \\
\hline Constante & $-64,7876$ & $* * *$ & $-78,2307$ & $-51,3444$ \\
Gasto Total com Inovação / Receita & $-31,7938$ & $* * *$ & $-41,9239$ & $-21,6637$ \\
Variação Imobilizado & $-0,0031$ & - & $-1,0047$ & 0,9985 \\
Variação Intangível & $-22,2955$ & $* * *$ & $-34,1477$ & $-10,4432$ \\
Log-Gasto Total com Inovação & 5,3118 & $* * *$ & 4,2066 & 6,4169 \\
R-quadrado & & & & \\
Estatística F (4,248) & & 0,291426 & \\
P-valor (F) & & 25,49965 & \\
\end{tabular}

Nota: ${ }^{* *},{ }^{* *},{ }^{*}$ significam nível de significância de $99 \%, 95 \%$ e $90 \%$, respectivamente Fonte: Elaborado pelos autores (2019).

Tabela 5 - Resultados da Regressão com dados em painel para Risco Anual Médio

\begin{tabular}{lcccc}
\hline \multicolumn{4}{c}{ Usando 253 observações / 134 unidades de corte transversal } \\
\hline \multicolumn{4}{c}{ Variável dependente: RISCO ANUAL MÉDIO } \\
\hline & Coeficiente & Grau de Significância & Intervalo de Confiança (95\%) \\
\hline Constante & 3,9925 & - & $-0,8105$ & 8,7954 \\
Gasto Total com Inovação / Receita & $-24,5244$ & $* * * 25)=1,970$ \\
Variação Imobilizado & 0,6446 & $*$ & $-30,2797$ & $-18,7690$ \\
Variação Intangível & 1,2915 & - & $-0,1045$ & 1,3937 \\
Log-Gasto Total com Inovação & 1,7881 & $* * *$ & $-2,1407$ & 4,7238 \\
R-quadrado & & & 1,4671 & 2,1091 \\
Estatística F (4,248) & & 0,505125 & & \\
P-valor (F) & & 63,28418 & \\
\hline
\end{tabular}

Nota: ${ }^{* *},{ }^{* *},{ }^{*}$ significam nível de significância de $99 \%, 95 \%$ e $90 \%$, respectivamente Fonte: Elaborado pelos autores (2019).

Tabela 6 - Resultados da Regressão com dados em painel para Sharpe

\begin{tabular}{lcccc}
\hline \multicolumn{4}{c}{ Usando 253 observações / 134 unidades de corte transversal } \\
\hline & Variável dependente: SHARPE & \\
\hline & Coeficiente & Grau de Significância & $\begin{array}{c}\text { Intervalo de Confiança }(95 \%) \\
\text { T }(248 ; 0,025)=1,970\end{array}$ \\
\hline Constante & $-0,5104$ & $* * *$ & $-0,7485$ & $-0,2722$ \\
Gasto Total com Inovação / Receita & $-0,0180$ & - & $-0,1441$ & 0,1081 \\
Variação Imobilizado & $-0,0014$ & - & $-0,0087$ & 0,0059 \\
Variação Intangível & 0,0472 & - & $-0,0914$ & 0,1859 \\
Log-Gasto Total com Inovação & 0,0347 & $*$ & 0,0170 & 0,0524 \\
R-quadrado & & 0,0728 & & \\
Estatística F (4,248) & & 4,8667 & \\
P-valor (F) & & 0,0008 & \\
\hline
\end{tabular}

Nota: ${ }^{* * *},{ }^{* *},{ }^{*}$ significam nível de significância de 99\%, 95\% e 90\%, respectivamente Fonte: Elaborado pelos autores (2019). 


\section{DISCUSSÃO}

Pode-se dizer que o cenário brasileiro sofreu certa mudança no período analisado; possivelmente devido à alta quantia necessária para o investimento em imobilizado, como máquinas, equipamentos, veículos, entre outros componentes. As empresas procuram adotar uma nova atitude, buscando formas acessíveis de investimento, devido ao cenário de incerteza enfrentado pelo país durante o período de estudo.

A amostra apresentou uma volatilidade menor dos ativos em relação ao mercado (beta igual a 1), porém uma análise profunda do beta é arriscada, visto que algumas empresas não apresentaram nenhum valor para este índice - valores estes que foram substituídos pelo beta médio setorial - oferecendo grande margem para a ocorrência de falhas na análise.

O desvio-padrão das variáveis de risco, principalmente o índice de Treynor, pode ser considerado elevado e acaba confirmando a grande disparidade dos dados (heterogeneidade), o que tende a dificultar a padronização de comportamentos dessa variável, já que há pequenas e grandes empresas na amostra, não sendo possível, por exemplo, determinar com toda certeza que um determinado comportamento serve para ambos os tipos de empresa.

Como o impacto dos gastos na capacidade de inovar tem efeito negativo no desempenho dos ativos das empresas mensurados pelos índices Treynor e Sharpe, pode-se inferir que estes investimentos contribuem para um retorno inferior ao requerido pelos acionistas, considerando o risco (seja pelo beta - Treynor, como pela variância do ativo - Sharpe). Esse resultado pode ser reflexo da forma como esses recursos estão sendo investidos ou mesmo gerenciados, dada a complexidade do processo de inovação (SANTOS; BASSO; KIMURA, 2012).

O risco anual médio representa o risco tecnológico, empresarial e financeiro das empresas da amostra que, por sua vez, apresentou uma média positiva e significativa perante a média do beta, ou seja, de forma geral as empresas apresentaram um risco superior ao risco de mercado, seja devido ao nível de endividamento ou a problemas operacionais.

Ao incluir a base de dados no software GRETL foi aplicado logaritmo na variável gasto total com inovação com o intuito de preservar o padrão de dispersão dos dados, reduzindo-se assim os valores da variável, sem prejuízo ao padrão de variação (CARVALHO, 2015). Por isso, foi desconsiderado o diagnóstico a respeito dos valores inteiros da variável "gasto total com inovação", durante a análise dos modelos.

Em tese, ao realizar os testes e as regressões no software mencionado anteriormente, as análises deveriam ter sido feitas com 438 observações (dados de 3 anos de 146 empresas diferentes); porém, em função da ausência de informações completas para rodar o modelo (valores zerados em todas as variáveis da mesma empresa em determinado ano), o software GRETL, com o intuito de não prejudicar a regressão, utilizou somente 253 observações correspondentes a 134 empresas, deixando de lado 12 empresas da amostra, visto que estas não apresentaram os valores suficientes para a realização da análise.

Este fato corrobora com o cenário do período estudado, em que as empresas acabam optando por investimentos de baixo risco devido à crise política e econômica instaurada no país, atuando de forma conservadora perante seus ativos. A atitude conservadora em si não se caracteriza como um problema, mas a ausência de investimentos pode provocar efeitos negativos no desempenho organizacional, como a perda de oportunidades, por exemplo (BAÑOS-CABALLERO; GARCÍA-TERUEL; MARTíNEZ-SOLANO, 2014).

Enquanto o índice de Treynor apresentou uma correlação inversa apenas com o intangível e a parcela do gasto com inovação sobre a receita, o índice de Sharpe apresentou uma relação negativa apenas com o imobilizado, criando margem para constatações como: quanto maior o investimento em imobilizado, pior o resultado decorrente do investimento; e quanto maior o investimento em intangível, menor o retorno relativo consumido pelo risco de mercado.

Para Schumpeter (1927), somente grandes empresas poderiam ter inovação, porque teriam maior capacidade de investimento; no entanto, se o impacto do volume de investimento em inovação no risco é positivo e significativo, mostra que o tamanho e o volume do investimento em inovação podem trazer maior 
incerteza aos investidores quanto à qualidade destes investimentos, enquanto um reflexo de problemas de agência.

Por outro lado, o indicador que relaciona o investimento em inovação com a receita mostrou impacto negativo e significativo na volatilidade, assinalando que os investimentos em inovação podem contribuir para um menor risco na empresa. Essa contradição inicial entre as duas variáveis sugere que os efeitos dos investimentos em inovação no risco não seguem um comportamento linear, conforme já apresentado no estudo de Amorim, Lima e Murcia (2012), sendo provável que o efeito seja uma parábola, assim os investimentos em inovação gerariam uma redução no risco até certo ponto, quando então, um maior volume de investimentos poderia ocasionar um efeito inverso no risco.

Cabe ressaltar que a análise do tamanho da influência dos coeficientes pode não condizer com a realidade, visto que há restrições quanto à normalidade das variáveis, ausência de normalidade dos resíduos e ainda o efeito significativo da constante que representa as variáveis que influenciam e que não foram analisadas no modelo, sendo, por esta razão, que a análise dos modelos gira em torno do sinal do coeficiente, do grau de significância, dos intervalos de confiança e das análises do coeficiente de determinação $\left(R^{2}\right)$ e do $p$ valor da estatística F, citados na pesquisa de Gujarati (2006).

Ao analisarmos o modelo com a variável dependente Beta, vale destacar que as variáveis gasto total com inovação sobre receita e logaritmo gasto total com inovação apresentaram significância e demonstraram capacidade de influenciar nas variáveis de risco. $O$ fato de a variável constante apresentar alto grau de significância no modelo, ocorre visto que é coerente imaginar que existem outras variáveis que também influenciam no risco das empresas.

No modelo 3, a significância e o efeito positivo do volume de investimentos em inovação no índice Treynor sugerem a importância do tamanho da empresa e sua capacidade de investimento em inovação para gerar excesso de retorno frente o risco sistemático. A importância do tamanho do investimento em inovação para gerar um desempenho positivo e superior ao mercado encontra os pressupostos de Schumpeter (1927) quanto ao efeito do tamanho da empresa para a inovação, em função da sua maior capacidade de levantar recursos, maior disponibilidade de capital humano e aprendizagem organizacional.

Já os outros coeficientes são significativos e influenciam de fato na variável dependente de forma inversa, sendo possível afirmar que quanto maior o montante investido menor tem sido o retorno adquirido em relação ao mercado - esse fato ocorre visto que a quantidade investida em determinado investimento não garante sempre um retorno satisfatório, devido à incerteza presente nesses casos (SANTOS; BASSO; KIMURA, 2012).

Além disso, vale destacar que ao investir a empresa está optando por realocar determinada quantidade de recursos na busca por uma possível rentabilidade futura ao invés de manter o montante em caixa parado, em uma tentativa de criar valor à empresa - tentativa esta que está suscetível a trazer resultados satisfatórios ou não devido ao grau de incerteza e a gama de variáveis influenciadoras presente (MENEZES et al., 2011).

Na tabela 6, enquanto o gasto total com inovação sobre a receita apresentou uma relação negativa, onde sob qualquer hipótese essa variável gera um efeito negativo no risco da empresa, a variável log-gasto total com inovação tem a capacidade de influenciar positivamente o risco da empresa. Sendo assim, através da análise das variáveis de maior significância é possível identificar que o montante destinado pela empresa para o investimento em inovação pode gerar efeitos positivos e negativos no risco da empresa, ou seja, deve haver outros fatores relevantes visto que cada empresa possui objetivos singulares e está inserida em um cenário específico sofrendo influência de fatores também específicos a ela.

Por último, realizou-se uma análise com o índice de Sharpe como variável dependente, onde novamente, a constante apresentou grau de significância elevado, influenciando negativamente na variável dependente sob qualquer hipótese. Sendo assim, através dessa tabela é possível perceber que o investimento em inovação tem a capacidade de interferir positivamente no desempenho e no retorno esperado pela empresa e, apesar disso, há outros fatores que não foram analisados e que influenciam no desempenho de um ativo. 
Esse cenário demonstra que o desempenho não sofre influências dos recursos alocados para o investimento em imobilizado ou intangível, nem da parcela da receita investida; porém, a simples alocação de recursos para o investimento em atividades inovativas já demonstrou um resultado inverso, sendo altamente significativa e influenciadora do comportamento da variável risco.

Sendo assim, de modo geral, é possível encontrar casos onde as variáveis de inovação exercem influência no risco e casos onde não exercem efeito nenhum no comportamento desta variável; fato este possível de ser identificado em todos os modelos analisados até aqui. Desse modo, assim como foi verificado no estudo de Amorim, Lima e Murcia (2012), é plausível a afirmação dos autores a respeito da falta de concordância na literatura quando analisamos o impacto entre inovação e risco, visto que há diversas teorias que encontram relação nula entre esses fatores e outras tantas que contradizem essa afirmação.

Vale destacar ainda que apenas um dos quatro modelos realizados pelo método dos mínimos quadrados ponderados não apresentou grau de significância plausível para a variável constante - que representa os fatores não analisados que podem influenciar na variável dependente - ou seja, entende-se que há outros fatores que influenciam a variável dependente e não somente a inovação.

Com o intuito de resumir as análises feitas anteriormente, a Tabela 8 foi elaborada pelo autor apresentando os principais resultados encontrados de forma condensada, contendo a direção e o grau de significância entre as variáveis de inovação e de risco.

Tabela 8 - Análise Conjunta

\begin{tabular}{ccccccc}
\hline & Constante & $\begin{array}{c}\text { Gasto Total } \\
\text { Inovação / } \\
\text { Receita }\end{array}$ & $\begin{array}{c}\text { Variação } \\
\text { Imobilizado }\end{array}$ & $\begin{array}{c}\text { Variação } \\
\text { Intangível }\end{array}$ & LN Gasto Inovação \\
\hline \multirow{2}{*}{ Beta } & Coeficiente & $(+)$ & $(+)$ & $(+)$ & $(+)$ & $(-)$ \\
Treynor & Significância & $* * *$ & $* *$ & - & - & $* * *$ \\
Risco & Coeficiente & $(-)$ & $(-)$ & $(-)$ & $(-)$ & $(+)$ \\
Anual & Coeficiente & $(+)$ & $(-)$ & $(+)$ & $(+)$ & $* * *$ \\
Médio & Significância & - & $* * *$ & $*$ & - & $(+)$ \\
Sharpe & Coeficiente & $(-)$ & $(-)$ & $(-)$ & $(+)$ & $(+)$ \\
\hline
\end{tabular}

Fonte: Elaborado pelos autores (2019).

Pode-se observar uma tendência da variável gasto total com inovação sobre receita em apresentar uma relação negativa com as variáveis de risco, enquanto a variável log-gasto total com inovação demonstrou ter uma relação positiva, na maioria dos casos, com o risco - contradição encontrada durante a análise que pode ser decorrente da instabilidade ou da forma de gestão das empresas, por exemplo. Já as outras variáveis não apresentaram um padrão significativo e de real predominância que mereça destaque.

De forma geral, o índice de Treynor (variável dependente) foi a que evidenciou o melhor resultado, visto que apresentou o maior número de significâncias entre as variáveis de inovação e risco. Entretanto, a análise que apresentou maior confiabilidade foi a realizada com o risco anual médio. Tanto a variável constante como a variável logaritmo do gasto total com inovação demonstraram-se significativas em três das quatro análises, caracterizando-se como variáveis de inovação que desempenham uma influência relevante na variável risco.

A variação do imobilizado foi a que apresentou o pior resultado ao analisarmos a influência gerada pela variável, visto que foi pouco relevante, e por vezes nem apresentou grau de significância. Contudo, cabe ressaltar que este estudo apresenta algumas limitações, como o curto período analisado, a falta de dados necessários disponíveis de algumas variáveis essenciais para o funcionamento do modelo, como o beta (risco de mercado), assim como a possibilidade de uma análise generalizada não coincidir com uma análise realizada por empresa. 


\section{CONCLUSÕES}

Este trabalho teve como objetivo avaliar o impacto dos investimentos na capacidade de inovar nos indicadores de risco de empresas brasileiras no período de 2012 a 2015 e, para tanto, foram coletadas informações de uma amostra de 146 empresas brasileiras listadas na bolsa brasileira no período de 2012 a 2015. Por meio das medidas estatísticas e dos indicadores de inovação e risco realizados é possível apontar que as variáveis de inovação podem influenciar tanto positiva como negativamente no comportamento das variáveis de risco analisadas. Ou seja, elas não seguem sempre o mesmo padrão de comportamento, razão pela qual diversos estudos na literatura que se contradizem, ora apresentando que as variáveis se relacionam, ora o contrário.

Pode-se concluir que o montante investido em inovação exerce influência com mais frequência do que quando analisado de forma separada através dos valores das contas imobilizado e intangível, visto que apresentou melhores resultados na análise realizada. Sendo assim, pode-se dizer que a variável inovação em si, impacta no risco, porém ao destrinchar essa variável, pode-se encontrar resultados diferentes.

Cabe ainda dizer que a análise da relação entre inovação e risco passa pelo gerenciamento dos recursos por parte da empresa, visto que os gestores têm a função de identificar todos os fatores que podem influenciar positiva ou negativamente suas decisões e seus investimentos. À medida que o investidor tem acesso a mais informações, quanto mais variáveis forem analisadas por ele e quanto maior for o período estudado, um resultado mais próximo da realidade será alcançado e, por consequência, a decisão tende a ser tomada de maneira mais simples e fácil pelo gestor.

Acredita-se que a presente pesquisa é relevante e contribui para a construção do conhecimento, sendo possível identificar implicações teóricas e administrativas que contribuem para o melhor gerenciamento dos recursos por parte das empresas, visto que na literatura há muitos estudos que verificam apenas a correlação das variáveis e poucas pesquisas com o objetivo de verificar o impacto das estratégias de inovação no risco.

Assim, a projeção para novos estudos compreende: i) Realização de uma análise do real efeito dos investimentos em inovação no desempenho das empresas (através de indicadores de liquidez, lucratividade, entre outros) utilizando o risco como moderador; e ii) Realização de uma análise por um período longo.

\section{REFERÊNCIAS}

AMORIM, A. L. C.; LIMA, I. S.; MURCIA, F. D.-R. Análise da relação entre as informações contábeis e o risco sistemático no mercado brasileiro. Revista de Contabilidade e Finanças - USP, São Paulo, v. 23, n. 60, p. 199-211, set./dez. 2012.

ARAÚJO, E. A. T.; OLIVEIRA, V. D. C.; SILVA, W. A. C. CAPM em estudos brasileiros: uma análise da pesquisa. Revista de Contabilidade e Organizações - USP, Ribeirão Preto, v. 6, n. 15, p. 95-122, 2012.

BAÑOS-CABALLERO, S.; GARCÍA-TERUEL, P.; MARTíNEZ-SOLANO, P. Working capital management, corporate perfomance and financial constraints. Journal of Business Research, v. 67, p. 322-328, 2014.

BARNEY, J.; WRIGHT, M.; KETCHEN Jr, D. J. The resource-based view of the firm: Ten years after 1991. Journal of Management, v. 27, p. 625-641, 2001.

BASSO, L. F. C.; KIMURA, H. O conceito de risco na Visão Baseada em Recursos (RBV): Uma análise exploratória. Revista de Administração do Mackenzie, São Paulo, v. 11, n. 5, p. 82-105, 2010.

BESANKO, D. et al. A Economia da estratégia. 3. ed. Porto Alegre: Bookman, 2006.

BOWERS, J.; KHORAKIAN, A. Integrating risk management in the innovation project. European Journal of Innovation Management, v. 17, n. 1, p. 25-40, 2014. 
CASSOL, A.; ZAPALAI, J.; CINTRA, R. F. Capacidade absortiva como propulsora da inovação em empresas incubadas de Santa Catarina. Revista de Ciências Administrativas, Fortaleza, v. 23, n. 1, p. 9-41, jan./abr. 2017.

CARVALHO, V. G. Influência das Informações Tributárias na Previsão dos Analistas Financeiros do Mercado de Capitais Brasileiro. 2015. 202 f. Tese (Doutor em Ciências Contábeis). - Universidade Federal do Rio Grande do Norte, Programa Multiinstitucional e InterRegional de Pós-Graduação em Ciências Contábeis, Natal, 2015.

COELHO, A. C. F.; CAMARA, S. F.; BRASIL, A. A coevolução das capacidades tecnológicas entre empresas multinacionais (MNE) e pequenas e médias empresas (PME) de tecnologia da informação e comunicação (TIC) em redes de tecnologia e inovação. Revista Ciências Administrativas, Fortaleza, v. 20, n. 2, p. 558-583, jul./dez. 2014.

CROSSAN, M. M.; APAYDIN, M. A multi-dimensional framework of organizational innovation: A systematic review of the literature. Journal of Management Studies, v. 47, n. 6, p. 1154-1179, 2010.

DAMODARAN, A. Avaliação de investimentos: Ferramentas e técnicas para a determinação do valor de qualquer ativo. 2. ed. Rio de Janeiro: QualityMark, 2010.

DANTHINE, J. P.; DONALDSON, J. B. Intermediate financial theory. 2nd. ed. San Diego: Elsevier, 2005.

ETGES, A. P. B. S.; CORTIMIGLIA, M. N. A systematic review of risk management in innovation-oriented firms. Journal of Risk Research, v. 22, n. 3, p. 364-381, 2019.

GODIN, B. A conceptual history of innovation. In: BATHELT, H.; COHENDET, P.; HENN, S.; SIMON, L. (org.). The elgar companion to innovation and knowledge creation. Cheltenham: Edward Elgar Publishing, 2017. p. 25-32.

GUJARATI, D. Econometria básica. São Paulo: Campus, 2006.

HAIR JR., J. F. et al. Análise multivariada de dados. 5. ed. Porto Alegre: Bookman, 2005.

HSIAO, C. Analysis of panel data. Cambridge: Cambridge University Press, 1986.

ILEVBARE, I. M.; PROBERT, D.; PHAAL, R. Towards risk-aware e roadmapping: Influencing factors and pratical measures. Technovation, v. 34, n. 8, p. 399-409, 2014.

JAFARI, M.; CHADEGANI, A. A.; BIGLARI, V. Effective risk management and company's performance: Investment in innovations and intellectual capital using behavioral and practical approach. Journal of Economics and International Finance, v. 3, n. 15, p. 780-786, 2011.

JIMÉNEZ-JIMÉNEZ, D.; SANZ-VALLE, R. Innovation, organizational learning, and performance. Journal of Business Research, v. 64, n. 4, p. 408-417 2011.

MARCELINO-SÁBADA, S. et al. Project risk management methodology for small firms. International Journal of Project Management, v. 32, n. 2, p. 327-340, 2014.

MARTIN, N. C.; SANTOS, L. R. D.; DIAS FILHO, J. M. Governança empresarial, riscos e controles internos: a emergência de um novo modelo de controladoria. Revista Contabilidade \& Finanças - USP, São Paulo, v. 1, n. 34, p. 7-22, 2004.

MATTA, V. E. D.; OLIVA, F. L.; VASCONCELLOS, E. P. G. D. Identificação e tratamento de riscos no lançamento de um novo produto no mercado aeroespacial. Sociedade, Contabilidade e Gestão, Rio de Janeiro, v. 10, n. 2, p. 1-19, 2015. 
MENEZES, U. G. D. et al. Gestão da inovação para o desenvolvimento sustentável: Comportamento e reflexões sobre a indústria química. Revista de Administração e Inovação, São Paulo, v. 8, n. 4, p. 88-116, 2011.

MEROÑO-CERDÁN, A. L.; LÓPEZ-NICOLÁS, C.; MOLINA-CASTILLO, F. J. Risk aversion, innovation and performance in family firms. Economics of Innovation and New Technology, v. 27, n. 2, p. 189-203, 2018.

MIORANDO, R. F.; RIBEIRO, J. L. D.; CORTIMIGLIA, M. N. An economic-probabilistic model for risk analysis in technological innovation projects. Technovation, v. 34, n. 8, p. 485-498, 2014.

OCDE. Manual de Oslo: Proposta de diretrizes para coleta e interpretação de dados sobre inovação tecnológica. 3. ed. Brasília: FINEP, 2005.

OSORIO, F.; DUPONT, L.; CAMARGO, M.; PALOMINOS, P.; PEÑA, J. I.; ALFARO, M. Design and management of innovation laboratories: Toward a performance assessment tool. Creativity and Innovation Management, v. 28, n. 1, p. 82-100, 2019.

PANG, C.; WANG, Q.; LI, Y.; DUAN, G. Integrative capability, business model innovation and performance: Contingent effect of business strategy. European Journal of Innovation Management, v. 22. n. 3, p. 541561,2019

PRAJOGO, D. I. The strategic fit between innovation strategies and business environment in delivering business performance. International Journal of Production Economics, v. 171, p. 241-249, 2016.

RAMOS, A.; ZILBER, S. N. O Impacto do investimento na capacidade inovadora da empresa. Revista de Administração e Inovação, São Paulo, v. 12, n. 1, p. 303-325, 2015.

SANTOS, D. F. L. et al. Innovation efforts and performances of Brazilian firms. Journal of Business Research, v. 67, n. 4, p. 527-535, abril 2014.

SANTOS, D. F. L.; BASSO, L. F. C.; KIMURA, H. A Estrutura da Capacidade de Inovar das Empresas Brasileiras: uma proposta de construto. Revista de Administração e Inovação, São Paulo, v. 9, n. 3, p. 103-128, 2012.

SANTOS, D. F. L.; BASSO, L. F. C.; KIMURA, H. The trajectory of the ability to innovate and the financial performance of the Brazilian industry. Technological Forecasting And Social Change, v. 127, p. 258-270, 2018.

SANTOS, M.; SILVA, M. Teoria de precificação por arbitragem: um estudo empírico no setor bancário brasileiro. Revista FAE, Curitiba v. 12, n. 1, p. 55-67, 2009.

SANTOS, J. O. D.; FONTES, R. J. D. S. Análise da relação entre o coeficiente beta, o índice de alavancagem d/e e a taxa de retorno de ações ordinárias de uma amostra de empresas listadas no lbovespa. Revista Contabilidade Vista \& Revista, Belo Horizonte, v. 22, n. 4, p. 173-197, 2011.

SCHUMPETER, J. A. The explanation of the business cycle. Economica, v. 21, p. 286-311, 1927.

SHAFIQUE, M. Thinking inside the box? Intellectual structure of the knowledge base of innovation research (1988-2008). Strategic Management Journal, v. 34, p. 62-93, 2013.

SILVA, F. B.; SANTOS, D. F. L.; RODRIGUES, S. V. Desempenho comparativo das ações de empresas com governança corporativa entre os setores bancário e industrial. Revista do Mestrado Profissional em Administração da Universidade Potiguar, Natal, v. 8, n. 2, p. 48-65, 2016.

THEISS, V. et al. Ranking do Índice Brasil de Inovação (IBI) nas atividades industriais e extrativas da região sul do Brasil. Revista de Administração e Inovação, São Paulo, v. 11, n. 3, p. 79-100, 2014.

VERGARA, S. C. Métodos de pesquisa em administração. 6. ed. São Paulo: Saraiva, 2015 
WU, D. D. et al. Modeling Technological innovation risks of an entrepreneurial team using system dynamics: An agent-based perspective. Techonological Forecasting and Social Change, v. 77, n. 6, p. 857-869, 2010. 